\title{
Crystal structure of 2RS,5-diphenyl-8RS-3,5,7-triazatricyclo[6.5.1.0 $\left.{ }^{3,7}\right]$ - tetradeca-9,11,13-triene-4,6-dione, $\mathrm{C}_{23} \mathrm{H}_{19} \mathrm{~N}_{3} \mathrm{O}_{2}$
}

\author{
G. E. Gream, P. K. Kirkbride and E. R. T. Tiekink*,I \\ The University of Adelaide, Department of Chemistry, Australia 5005
}

Received April 20, 2002, accepted and available on-line July 16, 2002; CCDC-No. 1267/851

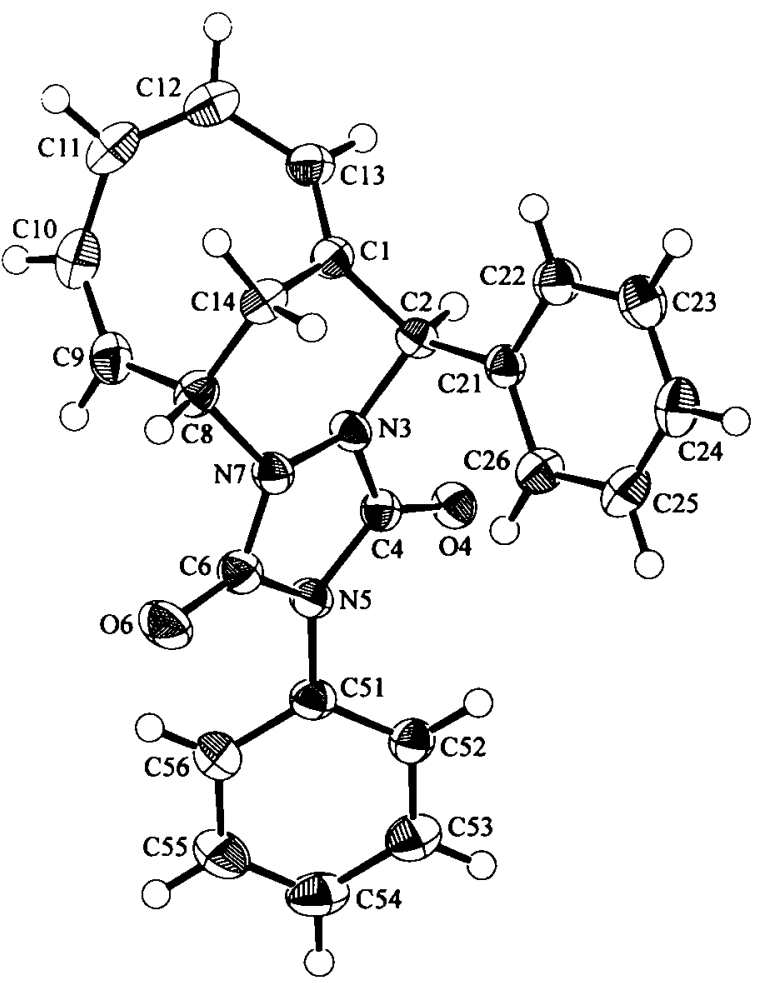

Abstract

$\mathrm{C}_{23} \mathrm{H}_{19} \mathrm{~N}_{3} \mathrm{O}_{2}$, monoclinic, $P 12{ }_{1} / n 1$ (No. 14), $a=10.247$ (2) $\AA$, $b=16.465(2) \AA, c=10.572(4) \AA, \beta=92.22(2)^{\circ}, V=1782.3 \AA^{3}$, $Z=4, R_{\mathrm{gt}}(F)=0.043, w R_{\mathrm{ref}}\left(F^{2}\right)=0.155, T=173 \mathrm{~K}$.

\section{Source of material}

Treatment of a mixture of $(E)$ - and (Z)-7-benzylidenecyclocta1,3,5-triene in ethyl acetate with 4-phenyl-1,2,4-triazoline-3,5dione gave a mixture of four compounds [1]. Chromatography on silica gel gave the title compound, mp $442 \mathrm{~K}-443 \mathrm{~K}$.

\section{Experimental details}

The C-bound $\mathrm{H}$ atoms were placed in their geometrically calculated positions and included in the final refinement in the riding model approximation.

\section{Discussion}

The central $\mathrm{C}_{2} \mathrm{~N}_{3}$ plane is planar to $0.042 \AA$ with the greatest deviation being $0.067(2) \AA$ for the $\mathrm{C} 6$ atom. The N5-phenyl group is not coplanar as evidenced by the C4-N5-C51-C52 torsion angle of $35(2)^{\circ}$. Presumably this occurs to as to minimise steric hin- drance. The molecule comprises a sequence of fused 5-, 6- and 8-membered rings. The sequence of $d(\mathrm{C}-\mathrm{C})$ bond distances for C8, C9 through to $\mathrm{C} 1$ are $1.483(4) \AA, 1.325(4) \AA, 1.446(4) \AA$, 1.323(4) $\AA, 1.441$ (3) $\AA$ and 1.322(3) $\AA$, respectively. The torsion angles $\mathrm{C} 8-\mathrm{C} 9-\mathrm{C} 10-\mathrm{C} 11\left(6.8(5)^{\circ}, \mathrm{C} 9-\mathrm{C} 10-\mathrm{C} 11-\mathrm{C} 12\right.$ $\left(45.7(7)^{\circ}\right), \quad \mathrm{C} 10-\mathrm{C} 11-\mathrm{C} 12-\mathrm{C} 13 \quad\left(-5.4(4)^{\circ}\right)$ and $\mathrm{C} 1-\mathrm{C} 13-\mathrm{C} 12-\mathrm{C} 11\left(-48.1(4)^{\circ}\right)$ reveal significant buckling within the ring. The patterns of parameters just described are indicative of a lack of conjugation within the 8-membered ring.

Table 1. Data collection and handling.

\begin{tabular}{ll}
\hline Crystal: & $\begin{array}{l}\text { colourless plate, } \\
\text { size } 0.08 \times 0.40 \times 0.40 \mathrm{~mm}\end{array}$ \\
Wavelength: & Mo $K_{\alpha}$ radiation $(0.71069 \AA)$ \\
$\mu:$ & $0.90 \mathrm{~cm}^{-1}$ \\
Diffractometer, scan mode: & Rigaku AFC6R, $\omega / 2 \theta$ \\
$2 \theta_{\text {max: }}$ & $50.6^{\circ}$ \\
$N(h k l)_{\text {measured, }} N(h k l)_{\text {unique }}:$ & 3448,3261 \\
Criterion for $I_{\text {obs, }} N(h k l)_{\text {gt: }}:$ & $I_{\text {obs }}>2 \sigma\left(I_{\text {obs }}\right), 2315$ \\
$N(\text { param })_{\text {refined: }}$ & 253 \\
Programs: & teXsan [2], SHELXS-86 [3], \\
& SHELXL-97 [4], ORTEPI [5]
\end{tabular}

Table 2. Atomic coordinates and displacement parameters (in $\AA^{2}$ ).

\begin{tabular}{|c|c|c|c|c|c|}
\hline Atom & Site & $x$ & $y$ & $z$ & $U_{\text {iso }}$ \\
\hline $\mathbf{H}(2)$ & $4 e$ & 0.1111 & 0.2154 & -0.1627 & 0.032 \\
\hline $\mathbf{H}(8)$ & $4 e$ & 0.4330 & 0.1629 & 0.1569 & 0.038 \\
\hline$H(9)$ & $4 e$ & 0.5568 & 0.2350 & 0.0019 & 0.043 \\
\hline $\mathbf{H}(10)$ & $4 e$ & 0.6282 & 0.1611 & -0.1398 & 0.051 \\
\hline $\mathbf{H}(11)$ & $4 e$ & 0.5658 & 0.0298 & -0.1873 & 0.050 \\
\hline$H(12)$ & $4 e$ & 0.3728 & 0.0028 & -0.2683 & 0.047 \\
\hline $\mathbf{H}(13)$ & $4 e$ & 0.2137 & 0.1132 & -0.2740 & 0.038 \\
\hline$H(14 a)$ & $4 e$ & 0.3597 & 0.0565 & 0.0176 & 0.037 \\
\hline$H(14 b)$ & $4 e$ & 0.2441 & 0.0958 & 0.0957 & 0.037 \\
\hline $\mathbf{H}(22)$ & $4 e$ & -0.0098 & 0.0742 & -0.0799 & 0.042 \\
\hline $\mathbf{H}(23)$ & $4 e$ & -0.1932 & 0.0453 & 0.0325 & 0.049 \\
\hline $\mathbf{H}(24)$ & $4 e$ & -0.2633 & 0.1356 & 0.1843 & 0.049 \\
\hline $\mathrm{H}(25)$ & $4 e$ & -0.1460 & 0.2531 & 0.2253 & 0.047 \\
\hline$H(26)$ & $4 e$ & 0.0383 & 0.2827 & 0.1127 & 0.040 \\
\hline H(52) & $4 e$ & 0.1023 & 0.4871 & 0.0472 & 0.040 \\
\hline $\mathbf{H}(53)$ & $4 e$ & 0.1146 & 0.6251 & 0.0858 & 0.049 \\
\hline$H(54)$ & $4 e$ & 0.3109 & 0.6834 & 0.1536 & 0.055 \\
\hline H(55) & $4 e$ & 0.4950 & 0.6038 & 0.1802 & 0.062 \\
\hline$H(56)$ & $4 e$ & 0.4871 & 0.4656 & 0.1353 & 0.054 \\
\hline
\end{tabular}

\footnotetext{
* Correspondence author (e-mail: chmtert@nus.edu.sg)

1 Current address: National University of Singapore,

Department of Chemistry, Singapore 117543
} 
Table 3. Atomic coordinates and displacement parameters (in $\AA^{2}$ ).

\begin{tabular}{|c|c|c|c|c|c|c|c|c|c|c|}
\hline Atom & Site & $x$ & $y$ & $z$ & $U_{11}$ & $U_{22}$ & $U_{33}$ & $U_{12}$ & $U_{13}$ & $U_{23}$ \\
\hline$O(4)$ & $4 e$ & $0.1284(2)$ & $0.37786(9)$ & $-0.1052(2)$ & $0.0317(9)$ & $0.0297(8)$ & $0.0322(9)$ & $0.0003(7)$ & $-0.0032(7)$ & $0.0035(7)$ \\
\hline$O(6)$ & $4 e$ & $0.4445(2)$ & $0.3287(1)$ & $0.2021(2)$ & $0.050(1)$ & $0.047(1)$ & $0.047(1)$ & $0.0062(9)$ & $-0.0235(9)$ & $-0.0101(9)$ \\
\hline$N(3)$ & $4 e$ & $0.2302(2)$ & $0.2642(1)$ & $-0.0281(2)$ & $0.029(1)$ & $0.025(1)$ & $0.0252(9)$ & $0.0003(8)$ & $-0.0053(8)$ & $0.0008(7)$ \\
\hline$N(5)$ & $4 e$ & $0.2859(2)$ & $0.3794(1)$ & $0.0597(2)$ & $0.029(1)$ & $0.025(1)$ & $0.031(1)$ & $0.0001(8)$ & $-0.0043(8)$ & $-0.0026(8)$ \\
\hline $\mathbf{N}(7)$ & $4 e$ & $0.3127(2)$ & $0.2476(1)$ & $0.0774(2)$ & $0.030(1)$ & $0.029(1)$ & $0.0276(9)$ & $0.0030(8)$ & $-0.0060(8)$ & $-0.0008(8)$ \\
\hline$C(1)$ & $4 e$ & $0.2387(2)$ & $0.1296(1)$ & $-0.0908(2)$ & $0.028(1)$ & $0.024(1)$ & $0.032(1)$ & $-0.0021(9)$ & $0.005(1)$ & $-0.0002(9)$ \\
\hline$C(4)$ & $4 e$ & $0.2051(2)$ & $0.3442(1)$ & $-0.0337(2)$ & $0.024(1)$ & $0.026(1)$ & $0.027(1)$ & $-0.0017(9)$ & $0.0024(9)$ & $0.0016(9)$ \\
\hline$C(6)$ & $4 e$ & $0.3587(2)$ & $0.3197(2)$ & $0.1227(2)$ & $0.035(1)$ & $0.035(1)$ & $0.031(1)$ & $0.002(1)$ & $-0.002(1)$ & $-0.003(1)$ \\
\hline$C(8)$ & $4 e$ & $0.3965(2)$ & $0.1751(1)$ & $0.0699(2)$ & $0.033(1)$ & $0.032(1)$ & $0.030(1)$ & $0.007(1)$ & $-0.000 \times 1)$ & $0.003(1)$ \\
\hline$C(9)$ & $4 e$ & $0.5073(2)$ & $0.1871(2)$ & $-0.0142(2)$ & $0.028(1)$ & $0.037(1)$ & $0.042(1)$ & $0.003(1)$ & $-0.003(1)$ & $0.004(1)$ \\
\hline$C(10)$ & $4 e$ & $0.5483(3)$ & $0.1418(2)$ & $-0.1083(3)$ & $0.032(1)$ & $0.053(2)$ & $0.044(2)$ & $0.006(1)$ & $0.006(1)$ & $0.008(1)$ \\
\hline$C(11)$ & $4 e$ & $0.5013(3)$ & $0.0701(2)$ & $-0.1747(2)$ & $0.046(2)$ & $0.042(2)$ & $0.037(1)$ & $0.016(1)$ & $0.012(1)$ & $0.003(1)$ \\
\hline$C(12)$ & $4 e$ & $0.3831(3)$ & $0.0515(2)$ & $-0.2206(2)$ & $0.050(2)$ & $0.034(1)$ & $0.035(1)$ & $0.005(1)$ & $0.011(1)$ & $-0.002(1)$ \\
\hline$C(13)$ & $4 e$ & $0.2692(2)$ & $0.1009(1)$ & $-0.2026(2)$ & $0.035(1)$ & $0.031(1)$ & $0.030(1)$ & $-0.004(1)$ & $0.005(1)$ & $-0.003(1)$ \\
\hline$C(21)$ & $4 e$ & $0.0322(2)$ & $0.1816(1)$ & $0.0050(2)$ & $0.025(1)$ & $0.030(1)$ & $0.025(1)$ & $0.0009(9)$ & $-0.0003(9)$ & $0.0014(9)$ \\
\hline $\mathrm{C}(22)$ & $4 e$ & $-0.0375(2)$ & $0.1113(2)$ & $-0.0175(2)$ & $0.034(1)$ & $0.035(1)$ & $0.037(1)$ & $-0.003(1)$ & $0.006(1)$ & $-0.001(1)$ \\
\hline$C(23)$ & $4 e$ & $-0.1461(3)$ & $0.0941(2)$ & $0.0489(3)$ & $0.034(1)$ & $0.042(2)$ & $0.048(2)$ & $-0.009(1)$ & $0.005(1)$ & $0.001(1)$ \\
\hline$C(24)$ & $4 e$ & $-0.1873(2)$ & $0.1471(2)$ & $0.1388(2)$ & $0.029(1)$ & $0.052(2)$ & $0.041(1)$ & $0.002(1)$ & $0.006(1)$ & $0.009(1)$ \\
\hline $\mathrm{C}(25)$ & $4 e$ & $-0.1185(3)$ & $0.2164(2)$ & $0.1623(2)$ & $0.038(1)$ & $0.044(2)$ & $0.037(1)$ & $0.011(1)$ & $0.010(1)$ & $0.001(1)$ \\
\hline$C(26)$ & $4 e$ & $-0.0089(2)$ & $0.2340(1)$ & $0.0956(2)$ & $0.035(1)$ & $0.030(1)$ & $0.035(1)$ & $0.001(1)$ & $0.007(1)$ & $0.000(1)$ \\
\hline $\mathrm{C}(51)$ & $4 e$ & $0.2931(2)$ & $0.4637(1)$ & $0.0856(2)$ & $0.032(1)$ & $0.028(1)$ & $0.030(1)$ & $-0.004(1)$ & $0.005(1)$ & $-0.003(1)$ \\
\hline$C(52)$ & $4 e$ & $0.1834(2)$ & $0.5110(2)$ & $0.0728(2)$ & $0.031(1)$ & $0.035(1)$ & $0.034(1)$ & $-0.001(1)$ & $0.006(1)$ & $-0.002(1)$ \\
\hline $\mathrm{C}(53)$ & $4 e$ & $0.1905(3)$ & $0.5923(2)$ & $0.0968(3)$ & $0.047(2)$ & $0.032(1)$ & $0.044(2)$ & $0.004(1)$ & $0.006(1)$ & $-0.002(1)$ \\
\hline$C(54)$ & $4 e$ & $0.3063(3)$ & $0.6268(2)$ & $0.1365(3)$ & $0.059(2)$ & $0.030(1)$ & $0.050(2)$ & $-0.006(1)$ & $0.012(1)$ & $-0.008(1)$ \\
\hline$C(55)$ & $4 e$ & $0.4150(3)$ & $0.5797(2)$ & $0.1515(3)$ & $0.048(2)$ & $0.044(2)$ & $0.065(2)$ & $-0.016(1)$ & $0.002(1)$ & $-0.015(1)$ \\
\hline$C(56)$ & $4 e$ & $0.4106(3)$ & $0.4980(2)$ & $0.1257(3)$ & $0.036(1)$ & $0.042(2)$ & $0.058(2)$ & $-0.005(1)$ & $-0.000(1)$ & $-0.012(1)$ \\
\hline
\end{tabular}

Acknowledgment. The Australian Research Council is thanked for support.

\section{References}

1. Kirkbride, P. K.: Some aspects of the chemistry of cyclooctatraene and its derivatives. Ph.D. Thesis. The University of Adelaide, Australia 1983.

2. teXsan: Single Crystal Structure Analysis Software. Version 1.04. Molecular Structure Corporation. The Woodlands, TX, USA 1997.

3. Sheldrick, G. M.: SHELX-86. Program for the automatic solution of crystal structures. University of Göttingen, Germany 1986.
4. Sheldrick, G. M.: SHELXI-97. Program for crystal structure refinement. University of Göttingen, Germany, 1997.

5. Johnson, C. K.: ORTEPII. Report ORNL-5138, Oak Ridge National Laboratory, Tennessee, USA 1976. 